\title{
Effects of Lipids on the Rheological Properties of Aqueous Soybean Protein Dispersions
}

\author{
Makoto MiUra and Fumio Yamauchi \\ Department of Food Chemistry, Faculty of Agriculture, \\ Tohoku University, Sendai 980, Japan
}

Received February 14, 1983

\begin{abstract}
The flow properties of soybean protein-lipid-water suspension systems and coagulated gels were related to a protein-lipid interaction. For powdered soybean lecithin-added soybean protein suspension systems and their heat-induced gels, the yield stress $\left(\sigma_{y}\right)$ and the consistency index $(K)$ increased with increasing amounts of added lipid, but the flow behavior index $(n)$ and the thixotropy index $(T I)$ decreased. On the other hand, there were only small changes in the magnitudes of the thixotropic parameters and the viscometric parameters $\left(\sigma_{y}, K\right.$ and $\left.n\right)$ after adding soybean oil at various concentrations to soybean protein dispersions. These facts suggested that the formation of a protein-phospholipid complex increased the effective particle size, and that the intermolecular entanglements and linkages among the protein molecules or among the proteinphospholipid complexes were weakened by the addition of polar lipids. The thixotropy index defined in this study is available for characterizing the stress decay that occurs within soybean protein dispersions and heat-induced gels as they are sheared.
\end{abstract}

Food systems are generally complex, particularly, in protein rich foods, proteins interact with oil and fats, carbohydrates and other compounds, and affect the physicochemical properties of the foods. It is necessary for the prediction of fundamental properties of the protein to determine the particular physicochemical states and interaction of the protein that result in the desired function.

The effects of lipids on the thermal gelation of soybean proteins have been studied by many investigators. Catsimpoolas and Meyer ${ }^{1,2)}$ showed that the inclusion of lipid materials enhanced the gelation of a soybean globulin. The texture of a soybean protein gel to which palm oil powder ${ }^{3)}$ or lecithin ${ }^{4)}$ was added, was reported by Yamano et al. Recently, Shimada et al. ${ }^{5)}$ investigated the hardness of soybean protein gels formed after adding unsaturated fatty acid methyl esters, triglycerides or edible oils. However, there is little information on the gel structure or its rheological properties. Viscosity is an experimentally measurable property that can yield information about the functional behavior as well as the physicochemical characteristics of the protein. Gelation is the physical manifestation of a protein denaturation process which is highly dependent upon the nature and amount of a protein, processing conditions, $\mathrm{pH}$, ionic environment, and coexistence of additives. Therefore, we have characterized the time-dependent flow properties of proteinlipid-water suspension systems for the establishment of relationships between structure and flow, and the correlation of rheological properties with protein-lipid interaction.

In this study, protein-lipid-water suspensions for viscometry were prepared by the addition of different lipids at several concentrations. Viscometric measurements were carried out in a cyclic shearing test and a cyclic temperature test by a modified concentric cylinder viscometer. ${ }^{6}$

\section{MATERIALS AND METHODS}

Materials. A soy protein isolate (Fujipurina Protein Co.) was used in these studies. It had the following proximate analysis: moisture $6.0 \%$, protein (nitrogen $\times$ 
6.25) $92.0 \%$, ash $5.0 \%$, crude fiber $0.3 \%$, crude fat $0.5 \%$ and $\mathrm{pH}$ of aqueous $10 \%$ suspension 7.0. Soybean oil was purchased from Nakarai Chemicals Co., Inc. A powdered soybean lecithin, which consists of $30 \sim 32 \%$ phosphatidylcholine, $22 \sim 28 \%$ phosphatidylethanolamine, $18 \sim 20 \%$ phosphatidylinositol, $3 \sim 4 \%$ phosphatidyserine, and $20 \sim 23 \%$ other phospholipids, was obtained from Tsurureshitin Kogyo Co.

All the reagents were of the highest grade.

Preparation of suspension systems. The buffer solution used here was a potassium phosphate buffer of 0.1 ionic strength contained $2.6 \mathrm{mM} \mathrm{KH} \mathrm{KH}_{2} \mathrm{PO}_{4}, 32.5 \mathrm{mM} \mathrm{K}_{2} \mathrm{HPO}_{4}$ and $4 \mathrm{mM} \mathrm{NaN}_{3}$ (PH 7.8).

Protein-lipid-water suspension systems were made at $20^{\circ} \mathrm{C}$ as follows. The protein was dispersed in the buffer, and degassed under vacuum. The soybean oil or the powdered soybean lecithin was added to the dispersions at various concentrations. The concentration of the protein was $12 \%(\mathrm{wt} / \mathrm{vol})$ and the concentrations of the added lipids were $0,0.5,1,2,4$ and $6 \%(\mathrm{wt} / \mathrm{vol})$.

Apparatus. The concentric cylinder viscometer used in the study was an Auto viscometer L type (Iwamoto Seisakusho Co.). This Couette type viscometer was modified by the addition of a temperature program controller. The inner cylinder (bob) and the rotating outer cylinder (cup) diameter were $2.8 \mathrm{~cm}$ and $3.0 \mathrm{~cm}$, respectively, and the immersion length was $7.0 \mathrm{~cm}$.

This viscometer was calibrated by using viscosity standards (JIS Z8809).

Viscometric measurements. Cyclic shearing tests and cyclic temperature tests were carried out for measuring the time-dependent flow properties and monitoring apparent viscosity changes of the suspension systems and the coagulated gels. After each sample had been introduced into the cup of the viscometer, it was exposed to violent stirring of $400 \mathrm{rpm}\left(\dot{\gamma}=649.9 \mathrm{sec}^{-1}\right)$ for $30 \mathrm{~min}$ to make the systems disperse well, and then allowed to rest for $30 \mathrm{~min}$ before beginning the tests so that it achieved thermal equilibrium and returned to its original state. Flow curves were recorded at $20^{\circ} \mathrm{C}$ by increasing the rotation velocity of the cup linearly from 30 to $150 \mathrm{rpm}$ over $10 \mathrm{~min}$ and then decreasing it back to $30 \mathrm{rpm}$ over another $10 \mathrm{~min}$. This was equivalent to an increase in the mean shear rate from 48.8 to $243.6 \mathrm{sec}^{-1}$ in $10 \mathrm{~min}$, with a corresponding time interval for the subsequent decrease in the mean shear rate to $48.8 \mathrm{sec}^{-1}$. Temperature effects on the apparent viscosity were determined under the shear rate of 81.2 $\sec ^{-1}$ by heating linearly from 20 to $90^{\circ} \mathrm{C}$ over $1 \mathrm{hr}$ and then cooling to $20^{\circ} \mathrm{C}$ over another $1 \mathrm{hr}$. Heat-induced gel was formed with protein thermo-denaturation during this cyclic temperature test.

Shear stress versus rotation velocity of the cup, and shear stress versus temperature were plotted directly on an $\mathrm{X}-\mathrm{Y}$ recorder. These series of tests were under direct digital control, and the obtained data were treated on a computer.

Viscometric data reduction. The approximate yield stress was obtained by the following flow model, that of Casson ${ }^{7}$ :

$$
\sqrt{\sigma}=K_{0}+K_{1} \sqrt{\dot{\gamma}}
$$

where $\sigma$ is the shear stress, $\dot{\gamma}$ is the shear rate, and $K_{0}$ and $K_{1}$ are constants. The magnitude of $K_{0}^{2}$ has been used as the yield stress by several workers. ${ }^{8)} K_{1}{ }^{2}$ is usually referred to as the limiting viscosity at an infinite rate of shear.

The obtained data in the down flow curves conform to the power law for suspending systems and coagulated gels. Its general form is:

$$
\sigma-\sigma_{y}=K \dot{\gamma}^{n}
$$

where $\sigma_{y}$ is the yield stress, $K$ is the consistency index, and $n$ is the flow behavior index. This power law model is known as the Herschel-Bulkley model.9)

The classical approach to characterizing structure breakdown during shear is the measurement of the hysteresis loop. ${ }^{10)}$ In this study, Equations 3 and 4 were used to describe the thixotropic flow behavior:

$$
\begin{gathered}
\Delta S=S t-S r \\
T I=\Delta S / S t
\end{gathered}
$$

where $S t$ and $S r$ are hysteresis loop areas defined in Fig. 1, $\Delta S$ is the area enclosed by the hysteresis loop, and $T I$ is the approximate index of thixotropy defined in this work.

Statistical data evaluation. The correlation analysis described below was performed separately for each lipidadded system. A matrix of correlation coefficients was computed from the amount of added lipid, the thixotropy index and the three viscometric parameters $\left(\sigma_{y}, K\right.$ and $\left.n\right)$. The computer program used was PFACTR (ACOS System/1000, ACOS-6 Time Sharing Library (TSS/LIB-6), NEC/Reference Manual).

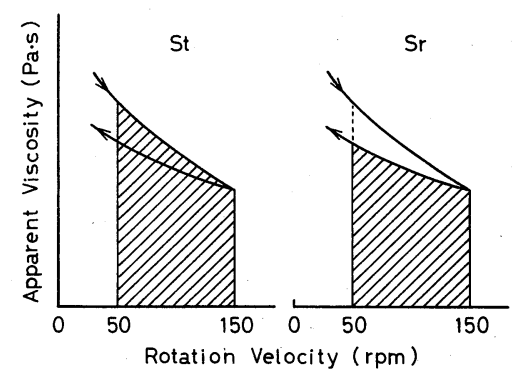

Fig. 1. Definition Areas of $S t$ and $S r$.

Shaded area under the up curve denotes $S t$ (left), and that under the down curve denotes $S r$ (right). 


\section{RESULTS}

Flow behavior for protein-lipid-water suspension systems

The shear-rate dependence of the apparent viscosity at $20^{\circ} \mathrm{C}$ for the soybean oil-added

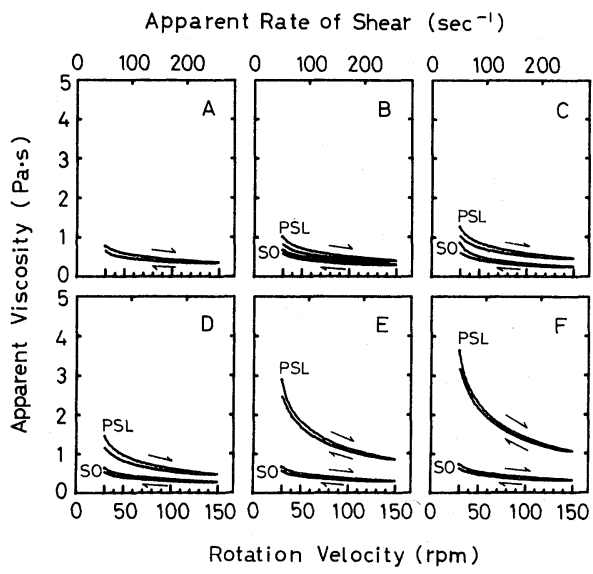

FIG. 2. Effect of Lipid Content on Hysteresis Curves of Protein-Lipid-Water Suspension Systems (12\% Protein, wt./vol.) at $20^{\circ} \mathrm{C}$.

Lipid content: without (A), 0.5\% (B), 1\% (C), 2\% (D), $4 \%$ (E), $6 \%$ (F). SO, soybean oil; PSL, powdered soybean lecithin.

TABle I. ThIXotropy INDEX AND VisCOMETRIC PARAMETERS OF ProteIN-LIPID-WATER SUSPENSION SYSTEMS

\begin{tabular}{ccccc}
\hline $\begin{array}{c}\text { Level of lipid } \\
\text { addition }(\%)\end{array}$ & $T I^{a}$ & $\begin{array}{c}\sigma_{y}{ }^{b} \\
(\mathrm{~Pa})\end{array}$ & $\begin{array}{c}K^{c} \\
\left(\mathrm{~Pa} \cdot \mathrm{s}^{n}\right)\end{array}$ & $n^{c}$ \\
\hline Without & 0.087 & 8.93 & 3.48 & 0.59 \\
Soybean oil & & & & \\
0.5 & 0.081 & 6.26 & 2.36 & 0.63 \\
1.0 & 0.093 & 8.30 & 3.23 & 0.58 \\
2.0 & 0.077 & 7.36 & 2.71 & 0.60 \\
4.0 & 0.079 & 6.97 & 2.59 & 0.63 \\
6.0 & 0.067 & 8.26 & 3.01 & 0.59 \\
Powdered lecithin & & & & \\
0.5 & 0.111 & 10.68 & 3.76 & 0.57 \\
1.0 & 0.087 & 18.04 & 6.88 & 0.52 \\
2.0 & 0.091 & 30.30 & 12.43 & 0.42 \\
4.0 & 0.043 & 69.70 & 32.71 & 0.33 \\
6.0 & 0.058 & 96.49 & 46.24 & 0.32 \\
\hline
\end{tabular}

a The approximate index of thixotropy defined in Eq. 4.

$b$ The yield stress in the Casson flow model (Eq. 1).

c The consistency index $(K)$ and the flow behavior index $(n)$ in the Herschel-Bulkley flow model (Eq. 2). suspension systems are shown in Fig. 2. In the shear rate range of 48.8 to $243.6 \mathrm{sec}^{-1}$, the apparent viscosity of all the suspensions decreased with the increase in shear rate. All the systems showed non-Newtonian flow behavior and thixotropy. The soybean oil seemed not to affect the shapes of the hysteresis loops. The thixotropy index and the viscometric parameters for these suspension systems are given in Table $I$. The values of the thixotropy index $(T I)$, the yield stress $\left(\sigma_{y}\right)$, the consistency index $(K)$ and the flow behavior index $(n)$ slightly fluctuated as the amount of the added soybean oil increased. The correlation analysis was further performed for both the thixotropy index and the viscometric parameters. Although all the parameters were not significantly correlated with the level of added lipid, the flow behavior index was significantly negatively correlated with the consistency index $(r=-0.940)$ and the yield stress $(r=-0.961)$. The correlation of the consistency index to the yield stress was significant and positive $(r=0.994)$.

For the powdered lecithin-added suspension systems, apparent viscosity decreased with an increase in the shear rate (Fig. 2). All the suspensions showed thixotropic flow behavior. Apparent viscosity increased as the amount of the added lipid increased. This tendency was noticeable at higher levels $(>3 \%)$ of lipid addition (Figs. 2E and F). However, the hysteresis loop area became smaller above 5\% powdered lecithin. As shown in Table I, the thixotropy index decreased with increasing amounts of added lipid. The consistency index and the yield stress considerably increased as the level of the lipid addition increased. In contrast, the flow behavior index decreased. All the parameters were significantly correlated with the amount of lipid. For the yield stress and the consistency index, the correlations with the level of addition were large ( $r=0.971$ and $r=0.969$, respectively). The correlations of the flow behavior index $(r=$ $-0.848)$ and the thixotropy index $(r=-0.755)$ to the amount of lipid were significant and negative. 


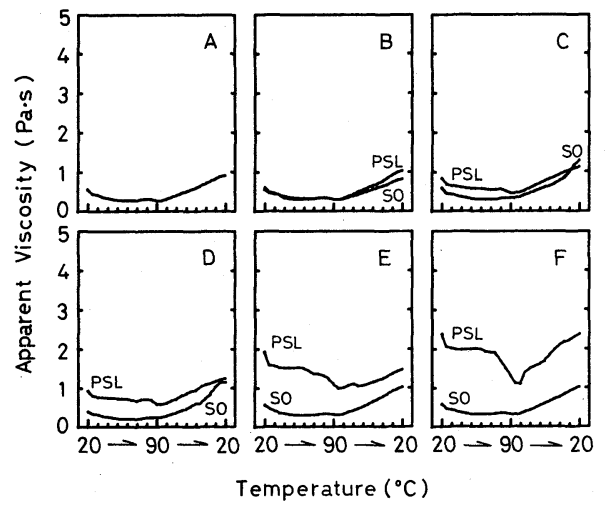

Fig. 3. Apparent Viscosity at the Shear Rate of 81.2 $\mathrm{sec}^{-1}$ as a Function of Temperature $\left( \pm 70^{\circ} \mathrm{C} / 1 \mathrm{hr}\right)$ of Protein-Lipid-Water Suspension Systems $(12 \%$ Protein, wt./vol.).

Lipid content: without (A), $0.5 \%$ (B), $1 \%$ (C), $2 \%$ (D), $4 \%$ (E), $6 \%$ (F). SO, soybean oil; PSL, powdered soybean lecithin.

\section{Heat-induced gel forming property}

The apparent viscosity was measured as a function of both heating and cooling temperatures (Fig. 3), and the soybean oil-added systems behaved as follows. The apparent viscosity, generally, decreased with an increase in temperature in the range of about 20 to $70^{\circ} \mathrm{C}$ in the heating process. In spite of the viscosity increase in the range of about 70 to $80^{\circ} \mathrm{C}$, a viscosity decrease was observed at the final stage of the heating process from about 80 to $90^{\circ} \mathrm{C}$. The apparent viscosity of the system increased considerably during the cooling process.

The relations between the viscosity and the temperature during both heating and cooling processes for the powdered lecithin-added suspension systems were also measured, as shown in Fig. 3. The observed curves with lower amounts of lecithin $(<2 \%)$ were similar in shape to those of the soybean oil-added systems, but had higher values. On the other hand, the curves at higher levels of lecithin addition $(>3 \%)$ had different patterns in shape (Figs. 3E and F). At these levels, the apparent viscosity decreased with increasing temperature in the range of 20 to $70^{\circ} \mathrm{C}$, and considerably decreased with increasing tem-

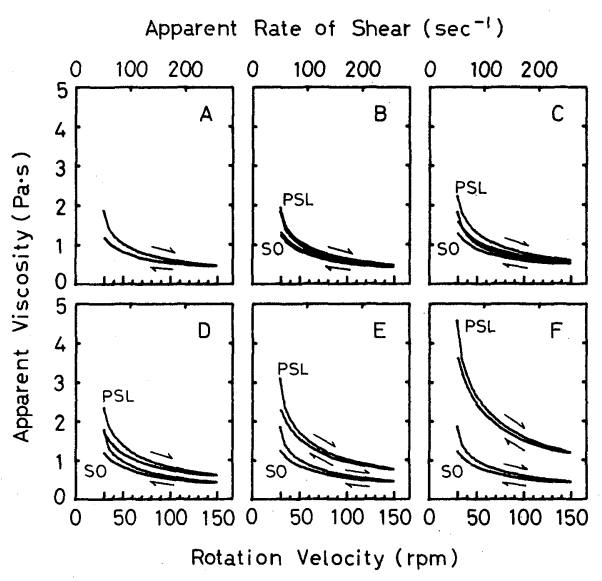

Fig. 4. Effect of Lipid Content on Hysteresis Curves of Lipid-added Gels at $20^{\circ} \mathrm{C}$.

Test conditions are same as in Fig. 2.

perature at the final stage of the heating range of about 70 to $90^{\circ} \mathrm{C}$. The increase of apparent viscosity was found in the cooling process. Moreover, the apparent viscosity of the systems with above $5 \%$ lipid added considerably increased during this process (Fig. 3F). The Weissenberg effect (normal-stress effect) ${ }^{11)}$ was observed for the suspensions at higher levels of lipid addition ( $>8 \%$, data not shown). Due to this effect, the suspending systems showed fluctuation in apparent viscosity during a long term $(<2 \mathrm{hr})$ shear deformation $\left(81.2 \mathrm{sec}^{-1}\right)$.

\section{Flow behavior for heat-induced gels}

After the cyclic temperature testing the resulting samples were used as the heat-induced gels in this measuring program. The effect of the shear rate on the apparent viscosity at $20^{\circ} \mathrm{C}$ for the gels is shown in Fig. 4. With a shear rate of 48.8 to $243.6 \mathrm{sec}^{-1}$, the apparent viscosity of all the gels decreased as the shear rate increased, and this decreasing tendency was noticeable at a lower shear rate. Larger hysteresis loops, which indicated higher apparent viscosity, were observed in all the gels. Table II presents the thixotropy index $(T I)$ and the viscometric parameters of the gels. Tr's of the soybean oil-added gels fluctuated, and the consistency index $(K)$, the flow behavior index $(n)$, and the yield stress $\left(\sigma_{y}\right)$ remained constant. 
TABLE II. Thixotropy InDEX AND Viscometric Parameters of LiPID-ADDED Gels

\begin{tabular}{ccccc}
\hline $\begin{array}{c}\text { Level of lipid } \\
\text { addition }(\%)\end{array}$ & $T I$ & $\begin{array}{c}\sigma_{y} \\
(\mathrm{~Pa})\end{array}$ & $\begin{array}{c}K \\
\left(\mathrm{~Pa} \cdot \mathrm{s}^{n}\right)\end{array}$ & $n$ \\
\hline Without & & & & \\
Soybean oil & 0.108 & 29.27 & 12.43 & 0.40 \\
0.5 & 0.081 & 29.70 & 12.87 & 0.39 \\
1.0 & 0.072 & 28.05 & 11.62 & 0.37 \\
2.0 & 0.068 & 29.68 & 12.46 & 0.41 \\
4.0 & 0.081 & 29.64 & 12.67 & 0.39 \\
6.0 & 0.090 & 31.53 & 13.85 & 0.37 \\
& & & & \\
Powdered lecithin & & & & \\
0.5 & 0.101 & 29.58 & 12.78 & 0.39 \\
1.0 & 0.091 & 37.11 & 15.74 & 0.40 \\
4.0 & 0.090 & 43.47 & 19.66 & 0.35 \\
6.0 & 0.062 & 73.44 & 33.59 & 0.34 \\
& 0.052 & 119.47 & 55.49 & 0.33 \\
\hline
\end{tabular}

For the thixotropy index and the viscometric parameters, see footnote to Table I.

All the parameters of the gels were not significantly correlated with the amount of the lipid. However, the consistency index was significantly and positively correlated with the yield stress $(r=0.949)$, whereas it was negatively correlated with the flow behavior in$\operatorname{dex}(r=-0.622)$.

For the powdered lecithin-added gels, apparent viscosity also increased with an increase in the amount of the lipid (Fig. 4). However, the hysteresis area became smaller at higher levels $(>3 \%$ ) of lipid addition (Figs. 4E and F). Although $T I$ considerably decreased with increasing amounts of lipid, the consistency index and the yield stress considerably increased (Table II). The flow behavior index slightly decreased and it was lowest at the $8 \%$ level (data not shown). All the parameters were significantly correlated with the amount of added lipid. For the consistency index and the yield stress, the correlations with the amount of lipid were positive and of large magnitude ( $r=0.888$ and $r=0.925$, respectively). The correlations of the flow behavior index and $T I$ to the level of the lipid addition were significant and negative $(r=-0.759$ and $r=-0.896$, respectively).

\section{DISCUSSION}

Effects of protein-lipid interaction on soy protein gelation have been partially characterized. ${ }^{2)}$ However, it is difficult to standardize the measurement of functional behavior, to generalize the findings of a certain analytical method, and to use the measured results from one system to predict the behavior in another. Measurements of the functional properties that have general applicability must be based upon the fundamental physicochemical properties of the components. ${ }^{12)}$ Therefore, we studied rheological properties of soybean protein dispersions in the presence or absence of neutral or polar lipids.

The thixotropy index defined in this study is available for characterizing the stress decay, which is due to structural changes with time that occur in the suspending system as it is sheared. The thixotropy indices, which were calculated from the flow curves, were significantly correlated with the flow behavior indices (correlation coefficients ranging from 0.588 to 0.730 , significant at the $5 \%$ level). The flow behavior index is unity in the case of a Newtonian flow and is used as a measure of the deviation from Newtonian flow behavior. For pseudoplastic fluids, the magnitude of the flow behavior index is less than unity; the smaller its magnitude the greater is the shearthinning flow behavior. ${ }^{8)}$ Moreover, the thixotropy index can be obtained easily from the hysteresis loops.

The dispersed systems of soybean protein, lipids, and water indicated a flow behavior having a shear-sensitive and time-dependent character, namely thixotropy. Only small variations of the flow properties were observed among the neutral lipid-added systems. The adsorption of the proteins on soybean oil droplets resulted in the formation of emulsions. However, the formation of emulsions may not change the effective particle size of the dispersed globules in the systems. For the polar lipid-added systems, the consistency index and the yield stress increased with increasing amounts of added lipid. This fact means 
that the mechanical consistency of the systems increased by addition of the phospholipid to the system. Ohtsuru et al. ${ }^{13)}$ reported that phosphatidylcholine was associated with soybean protein through either hydrophobic interaction between a phosphatidylcholine molecule and the protein, or the binding of phosphatidylcholine lamellae to the protein surface. Therefore, it may be also concluded in this investigation that phospholipids interacted with the protein by the mechanisms described above. The formation of a proteinphospholipid complex may increase the viscosity of the dispersed system, as it changes the effective particle size and the volume of the dispersed phase. The thixotropy index and the flow behavior index decreased with increasing amounts of lipid. These facts suggest that the entanglements and linkages among proteinphospholipid complexes are weakened by the addition of phospholipids to the system. The viscosity of the lipid itself was negligibly small within the concentration range used. The gentle decrease at the range from 20 to $70^{\circ} \mathrm{C}$ in the heating process suggested that a structure breakdown occurred in the system. Thus the temperature-thinning effect, which is typical for suspension systems of macromolecules, contributed to this flow behavior. In the range of 70 to $80^{\circ} \mathrm{C}$, the apparent viscosity increased with increasing temperature which indicates that the protein is unfolded by heat treatment. Umeya et $a l .{ }^{14)}$ reported that the apparent viscosity of the aqueous suspension system of the acid-precipitated protein (APP) of soybean increased at the final stage of heating in the range of about 70 to $90^{\circ} \mathrm{C}$, but we obtained different results in this range. At the end of the heating process, the apparent viscosity decreased rather than increased as can be seen in the APP, which suggests that structure breakdown is predominant over the building up of three dimensional intermolecular networks in the dispersed systems of soy protein isolate used in this study. This phenomenon became remarkable upon the addition of the powdered lecithin to the dispersed systems. It seems likely that the binding of phospholipids to the protein surface weaken the intermolecular entanglements and linkages between protein molecules in this temperature region. Thus the polar lipids may play significant roles in the structural changes including entanglement ${ }^{15}$ ) of protein molecules in dispersions.

Acknowledgments. We express our thanks to Fujipurina Protein Co. for kindly supplying soy protein isolate. We also thank Tsurureshitin Kogyo Co. for kindly supplying powdered lecithin.

\section{REFERENCES}

1) N. Catsimpoolas and E. W. Meyer, Cereal Chem., 47, 559 (1970).

2) N. Catsimpoolas and E. W. Meyer, Cereal Chem., 48, 159 (1971).

3) Y. Yamano, E. Miki and Y. Fukui, Nippon Shokuhin Kogyo Gakkaishi, 28, 131 (1981).

4) Y. Yamano, K. Sakamoto, J. Sonobe and E. Miki, Nippon Shokuhin Kogyo Gakkaishi, 28, 650 (1981).

5) K. Shimada and S. Matsushita, Agric. Biol. Chem., 45, 2877 (1981).

6) J. Umeya, F. Yamauchi and K. Shibasaki, Nippon Shokuhin Kogyo Gakkaishi, 27, 459 (1980).

7) N. Casson, "A Flow Equation for Pigment-Oil Suspensions of the Printing Ink Type. In Rheology of Disperse Systems," ed. by C. C. Mill, Pergamon Press, New York, 1959, p. 84.

8) M. A. Rao, J. Texture Studies, 8, 135 (1977).

9) P. Sherman, "Industrial Rheology," Academic Press, New York, 1970.

10) P. I. Figoni and C. F. Shoemaker, J. Texture Studies, 12, 287 (1981).

11) K. Weissenberg, Nature, 159, 310 (1947).

12) J. L. Shen, "Protein Functionality in Foods," ed. by J. P. Cherry, Southern Regional Research Center, Washington, D.C., 1981, p. 89.

13) M. Ohtsuru, M. Kito, Y. Takeuchi and S. Ohnishi, Agric. Biol. Chem., 40, 2261 (1976).

14) J. Umeya, F. Yamauchi and K. Shibasaki, Agric. Biol. Chem., 44, 1321 (1980).

15) W. W. Graessley, Adv. Polym. Sci., 16, 1 (1974). 